\title{
Comparação de dois métodos de controle de nematódeos gastrintestinais em borregas e ovelhas de corte
}

\section{Comparison of two methods for the control of gastrointestinal nematodes in lambs and ewes}

\author{
Maria Isabel Botelho Vieira ${ }^{1 *}$; Hélio Carlos Rocha²; Luiz Augusto Brandão Ractz \\ Rogério Nadal ${ }^{4}$; Roberto Bagatini de Moraes ${ }^{4}$; Iali da Silva Oliveira ${ }^{4}$
}

\section{Resumo}

Este trabalho teve como objetivo avaliar o controle de helmintos gastrintestinais em ovinos de corte em um sistema de pastoreio Voisin em quatro estações do ano. Foi realizado no Centro de Pesquisa Agropecuária da Universidade Passo Fundo no período de junho de 2005 a julho de 2006. Foram utilizados 84 fêmeas das raças Suffolk, Ile de France puras e mestiças as quais foram subdivididas em quatro grupos composto de 21 animais cada. Os grupos foram designados como G1 (ovelha tradicional), G2 (borrega tradicional) tratadas a cada 60 dias com Levamisole; os grupos G3 (ovelha Famacha ${ }^{\circledR}$ ), e G4 (borrega Famacha ${ }^{\circledR}$ ) foram avaliadas pelo método Famacha ${ }^{\circledR}$. As variáveis analisadas foram peso vivo e ovos por grama de fezes $(\mathrm{OPG})$. Em relação ao peso vivo não houve diferença estatística $(\mathrm{P}>0,05)$ entre borregas e ovelhas dos grupos tradicionais quando comparadas aos do método Famacha ${ }^{\circledR}$. Ocorreram diferenças no OPG no decorrer das estações do ano para as borregas. Houve uma significativa redução na freqüência dos tratamentos pelo método Famacha ${ }^{\circledR}$ quando comparado ao método tradicional, de 90,39\% para as borregas e 90,93\% para as ovelhas, tornando este método uma alternativa viável.

Palavras-chave: Ovinos, peso vivo, Famacha ${ }^{\circledR}$, Haemonchus contortus, OPG

\begin{abstract}
The objective of this work was to evaluate the control of nematode gastrintestinal on ovine meat breeds in Voisin pasturing system during one grazing year. The experiment was carried out at the Research Center Farm of the Passo Fundo University during the period of June 2005 to July 2006. The animals were kept in 85 pasture blocks with 810 square meters each. In the experiment 42 ewe hoggets and 42 Suffolk, Ile de France purebreed and crosses ewes were subdivided in four groups composed of 21 animals each. The groups had been assigned as G1 (traditional sheep), G2 (traditional young ewes) both treated every 60 days with Levamisole; the groups G3 (Famacha ${ }^{\circledR}$ sheep)=and G4 (Famacha ${ }^{\circledR}$ young ewes) were evaluated by the Famacha ${ }^{\circledR}$ method. The following variables were evaluated: live weight and worm egg counts (EPG). In relation to the live weights it was proven that it did not have statistics difference $(\mathrm{P}>0,05)$ between ewe hoggets and adult sheep of the traditional groups when compared with the ones of the Famacha ${ }^{\circledR}$ method. Differences in EPG counts during of the year in the ewe hoggets were found it had a significant reduction in the frequency of treatments for the Famacha ${ }^{\circledR}$ method when compared with the traditional method, of $90,39 \%$ for ewe hoggets and $90.93 \%$ for the sheep, becoming this method a viable alternative.
\end{abstract}

Key words: Sheep, live weight, Famacha ${ }^{\circledR}$, Haemonchus contortus, EPG

1 Professora do Curso de Medicina Veterinária da Universidade de Passo Fundo, RS, Brasil, Campus I, Bairro São José, BR 285, Km 171, Hospital Veterinário, CEP 99001-970, Passo Fundo, RS. E-mail: marisabel@upf.br.

2 Professor do Curso de Agronomia e Medicina Veterinária da UPF, Passo Fundo, RS.

Aluno do Curso de Biologia da UPF, Passo Fundo, RS.

Alunos do Curso de Medicina Veterinária da UPF, Passo Fundo, RS.

Autor para correspondência 


\section{Introdução}

A ovinocultura é uma atividade largamente explorada nos países tropicais, visando à produção sustentada de carne, leite e peles. O interesse pela exploração de ovinos vem aumentando nos países desenvolvidos, onde o uso de tecnologias é usado com o objetivo de aumentar a produção. Entretanto, as endoparasitoses gastrintestinais se constituem no principal fator limitante para a produção de ovinos em todo o mundo, especialmente, nas regiões tropicais, onde os prejuízos econômicos são mais acentuados (VIEIRA; CAVALCANTE, 2003).

Os efeitos dos parasitas na saúde dos ovinos caracterizam-se, principalmente, pela anemia e hipoproteinemia, que podem resultar em morte dos animais. A anemia produzida pelos parasitas gastrintestinais, principalmente pelo Haemonchus contortus, tem sido objeto de estudo por diversos pesquisadores (ADANS, 1981). O H. contortus é predominante em diversas regiões do Brasil, onde é considerado o principal parasita de ovinos, causador de mortes em animais, devido à ingestão de sangue na região do abomaso do hospedeiro (MOLENTO et al., 2004).

Tradicionalmente, os produtores recorreram ao uso exclusivo de substâncias químicas para o controle dos endoparasitas de ovinos, estratégia que foi ineficiente devido a diversos fatores, entre eles a falta de critério técnico na aplicação dos fármacos (LANUSSE; PRICHARD, 1993). No Rio Grande do Sul, a prevalência de resistência anti-helmíntica nos rebanhos ovinos representa um grave problema para o controle eficiente das helmintoses gastrintestinais (ECHEVARRIA et al., 1996).

$\mathrm{Na}$ busca de alternativas ao uso indiscriminado de fármacos, Van Wyk et al. (1997) iniciaram alguns estudos com o objetivo de avaliar individualmente os animais do rebanho através do acompanhamento da coloração da conjuntiva. O método é baseado na coloração da mucosa ocular, identificando os animais que apresentam graus de parasitose elevada (sensíveis), leve(resilientes), e os parasitados que não expressam sinais clínicos (resistentes). Os mesmos autores associaram os valores do hematócrito com as diferentes colorações da conjuntiva, sendo que estas foram estabelecidas através do auxílio da computação gráfica, representando cinco graus de anemia. Também foi comprovado que os diferentes graus de anemia apresentaram correlação de 0,8 com grau de confiabilidade superior a 95\% (VAN WYK; MALAN; BATH, 1997).

Como forma de estudar uma nova alternativa ao controle da verminose ovina foi realizado o presente trabalho no Planalto Médio do Rio Grande do Sul, visando comparar os métodos tradicional e Famacha® no controle de Haemonchus contortus.

\section{Material e Métodos}

O trabalho foi realizado no Centro de Extensão e Pesquisa Agropecuária da Universidade de Passo Fundo (CEPAGRO), no período de junho de 2005 a julho de 2006. Foram utilizados 84 animais, sendo divididos pela idade fisiológica em borregas e ovelhas das raças Suffolk, Ile de France puras e mestiças.

\section{Teste de sensibilidade dos antiparasitários}

O controle antiparasitário utilizado nos animais experimentais seguiu o método tradicional, de acordo com a indicação de ovos por grama de fezes (OPG), ou a cada 60 dias. Por este motivo, antes do início do experimento foi realizado um teste de sensibilidade dos antiparasitários, seguindo a metodologia padrão de redução de OPG (WALLER, 1997). Baseado no resultado do teste, o produto que apresentou a melhor eficácia foi o Levamisole, sendo, portanto, o escolhido para ser utilizado nos grupos experimentais.

\section{Grupos experimentais}

Os ovinos foram manejados em uma área de $77.343,18 \mathrm{~m}^{2}$ sistematizada pelo Pastoreio Racional 
Voisin, onde foram implantados 85 piquetes de 810 $\mathrm{m}^{2}(30 \mathrm{~m} \times 27 \mathrm{~m})$, mais corredores e áreas de lazer. Os animais permaneciam no piquete de um a dois dias dependendo das condições climáticas. Todos os grupos permaneceram em um único rebanho pastoreando uma área composta de Pennisetum clandestinum, Trifolium repens, Paspalum notatum, Lolium multiflorum, Cynodon sp, Avena strigosa, Pensacola sp, e algumas plantas invasoras. Foram usados 84 ovinos divididos em quatro grupos de 21 animais cada. O grupo 1 foi denominado de ovelha tradicional, o grupo 2 de borrega tradicional, onde todos os animais foram tratados a cada 60 dias utilizando seis aplicações do antiparasitário Levamisole no decorrer de um ano, pois foi o produto que apresentou melhor eficácia (90\%) no teste de sensibilidade antiparasitária. $\mathrm{O}$ grupo 3 foi denominado de ovelha Famacha ${ }^{\circledR}$ e o 4 de borrega Famacha ${ }^{\circledR}$ e foram avaliados a cada 15 dias pelo método Famacha ${ }^{\circledR}$, e a indicação de tratamento foi baseada neste método.

\section{Exame clínico}

Nos animais dos grupos 3 e 4, a inspeção da conjuntiva dos animais foi realizada por técnicos devidamente treinados, em intervalos de 15 dias. Durante o período experimental os exames da conjuntiva foram realizados através da comparação de diferentes tonalidades, de vermelho-rosado até o branco pálido, representando os números de 1 a 5 , e comparados com o cartão Famacha ${ }^{\circledR}$ desenvolvido para utilização no campo.

\section{Colheita das fezes}

A colheita das fezes dos grupos foi realizada a cada 30 dias, sendo as mesmas retiradas diretamente da ampola retal dos animais e acondicionadas em sacos plásticos, devidamente identificados, e levados ao Laboratório de Doenças Parasitárias da Universidade de Passo Fundo onde foram acondicionadas e refrigeradas até a realização dos exames laboratoriais, que ocorreu no prazo de $48 \mathrm{hs}$ após a colheita das fezes.

O exame das fezes foi realizado através da técnica descrita por Gordon e Whitlock (1939) modificada, de uso comum para a contagem de OPG, conforme Ueno e Gonçalves (1998). A coprocultura de larvas foi realizada através da técnica de Roberts e Sullivan (1950) e as larvas infectantes foram colhidas, segundo técnica descrita por Ueno e Gonçalves (1998).

\section{Pesagem}

Todos os animais foram pesados a cada 30 dias para comparar a média de ganho de peso entre os grupos analisados para discutir o custo - beneficio do método Famacha ${ }^{\circledR}$.

\section{Análise estatística}

Os dados de ovos por grama de fezes (OPG) foram transformados em $\log _{10}(\mathrm{OPG}+10)$ com o objetivo de obter normalidade dos valores de OPG (KAWANO; YAMAMURA; RIBEIRO, 2001). $\mathrm{Na}$ análise de peso vivo e OPG foi aplicado o Teste-t, sendo as variâncias analisadas quanto a homocedasticidade e após comparadas dentro dos grupos: Famacha ${ }^{\circledR}$ x tradicional; e entre as estações do ano: inverno, primavera, verão e outono.

\section{Resultados e Discussões}

O acompanhamento no decorrer de um ano forneceu informações importantes quanto aos períodos de maior infecção parasitária. $\mathrm{Na}$ coprocultura o $H$. contortus foi o mais prevalente, seguido de Trichostrongylus spp. Ramos et al. (2004) no Planalto Catarinense identificaram $H$. contortus como o nematódeo mais prevalente nos meses de verão e outono. No entanto, no mesmo trabalho ficou demonstrado que esta espécie foi encontrado na maioria dos meses, a partir de que os autores concluíram que este é o principal parasita do abomaso de ovinos nesta região. 
As médias do peso vivo entre os grupos Famacha ${ }^{\circledR}$ e tradicional para as borregas e ovelhas nas diferentes estações do ano analisadas através do Teste-t, não mostraram diferença significativa $(\mathrm{P}>0,05)$. Kawano, Yamamura e Ribeiro (2001) também não encontraram diferenças estatísticas em relação ao peso vivo entre animais tratados e não tratados, embora outros autores demonstraram quedas no ganho de peso vivo entre 20 a $40 \%$
(ECHEVARRIA, 1988). A amplitude de variação ficou entre $7,6 \%$ a $12,4 \%$ para as borregas Famacha ${ }^{\circledR} ; 6,7 \%$ a $8,5 \%$ para o grupo borregas tradicional; $12,8 \%$ a $15,6 \%$ para as ovelhas do grupo Famacha ${ }^{\circledR}$ e $15,4 \%$ a $18,4 \%$ para as ovelhas do grupo tradicional, evidenciando que não houve perda de peso vivo $(\mathrm{P}>0,05)$ nos animais submetidos ao controle de $H$. contortus pelo método Famacha ${ }^{\circledR}$, conforme Tabela 1.

Tabela 1. Média e desvio-padrão e probabilidade estatística da variável peso vivo (kg) de borregas e ovelhas submetidas a dois métodos de controle de Haemonchus contortus durante um ano

\begin{tabular}{lccc}
\hline Estações do ano/categoria & Famacha ${ }^{\circledR}$ & Tradicional & $\mathrm{P}(\mathrm{T} \leq \mathrm{t})$ \\
\hline Inverno/borregas & $58,2 \pm 4,45$ & $58,3 \pm 4,99$ & 0,889071 \\
Primavera/borregas & $66,5 \pm 6,09$ & $65,5 \pm 4,45$ & 0,56133 \\
Verão/borregas & $67,8 \pm 8,43$ & $68,1 \pm 6,42$ & 0,91036 \\
Outono/borregas & $70,1 \pm 7,87$ & $70,5 \pm 5,23$ & 0,85902 \\
Inverno/ovelhas & $74,8 \pm 10,14$ & $75,1 \pm 12,69$ & 0,943612 \\
Primavera/ovelhas & $73,4 \pm 11,52$ & $76,9 \pm 14,19$ & 0,39695 \\
Verão/ovelhas & $76,7 \pm 10,84$ & $78,5 \pm 14,19$ & 0,63574 \\
Outono/ovelhas & $82,4 \pm 10,40$ & $84,6 \pm 13,08$ & 0,57847 \\
\hline
\end{tabular}

A verminose pode acometer animais de qualquer sexo e idade, no entanto, o problema é mais severo em cordeiros (COLDITZ et al., 1996), e em fêmeas no período do periparto (AMARANTE et al., 1992). O’Sullivan e Donald (1970), relatam que a condição nutricional dos animais tem grande influência na resistência contra as infecções por nematódeos. Estes mesmos autores, afirmam que no período do periparto as ovelhas se tornam mais susceptíveis às infecções por nematódeos gastrintestinais, o que provoca aumento no número de ovos eliminado nas fezes e, conseqüentemente, da contaminação da pastagem. Com relação ao aumento do número de OPG na comparação dos grupos tradicional e Famacha ${ }^{\circledR}$ (Tabela 2) ocorreu uma diferença estatística no OPG das borregas durante a primavera $(\mathrm{P}<0,01)$, provavelmente em decorrência do aumento da precipitação pluviométrica deste período (150 $\mathrm{mm}$ ) associado a uma média de temperatura de $20^{\circ} \mathrm{C}$ favoráveis ao desenvolvimento dos nematódeos trichostrongilídeos. Embora o número médio de OPG na primavera nas borregas tenha sido mais elevado, estes animais não apresentaram sinais clínicos de anemia, sendo que apenas 1,05\% dos animais foram tratados. Carneiro et al. (2006) apresentaram resultados mostrando que existe um nível de tolerância aos efeitos patogênicos do parasitismo, mesmo animais com OPG elevado não apresentaram perdas de peso vivo, resultados estes semelhantes ao do presente trabalho.

Nas ovelhas na comparação entre os mesmos tratamentos e períodos não houve diferença estatística para a variável OPG (P>0,05). Braga (1986) realizou estudos sobre as condições climáticas no processo de translação, que consiste na taxa de contaminação dos animais com formas parasitárias infectantes. $\mathrm{O}$ índice pluviométrico foi o principal fator responsável pelo aumento das infecções parasitárias ocorridas no período do outono quando as condições de precipitação pluviométrica oscilaram entre $150 \mathrm{a}$ 200mm, conforme apresentado na Figura 1. 
Tabela 2. Média e desvio-padrão e probabilidade estatística da variável OPG* de borregas e ovelhas submetidas a dois métodos de controle de Haemonchus contortus durante um ano

\begin{tabular}{lccc}
\hline Estações do ano/categoria & Famacha ${ }^{\circledR}$ & Tradicional & $\mathrm{P}(\mathrm{T} \leq \mathrm{t})$ \\
\hline Inverno/borregas & $1,299 \pm 0,464$ & $1,107 \pm 0,267$ & 0,123252 \\
Primavera/borregas & $2,025 \pm 0,857$ & $1,095 \pm 0,233$ & 0,00004088 \\
Verão/borregas & $2,107 \pm 0,832$ & $2,069 \pm 0,745$ & 0,882686 \\
Outono/borregas & $2,062 \pm 0,704$ & $1,177 \pm 0,941$ & 0,674394 \\
Inverno/ovelhas & $1,644 \pm 0,694$ & $1,565 \pm 0,629$ & 0,7172241 \\
Primavera/ovelhas & $1,322 \pm 0,494$ & $1,371 \pm 0,660$ & 0,794658 \\
Verão/ovelhas & $1,580 \pm 0,723$ & $1,608 \pm 0,567$ & 0,896831 \\
Outono/ovelhas & $1,851 \pm 0,720$ & $1,890 \pm 0,738$ & 0,88356 \\
\hline
\end{tabular}

Variável sob transformação de $\log (\mathrm{x}+10)$

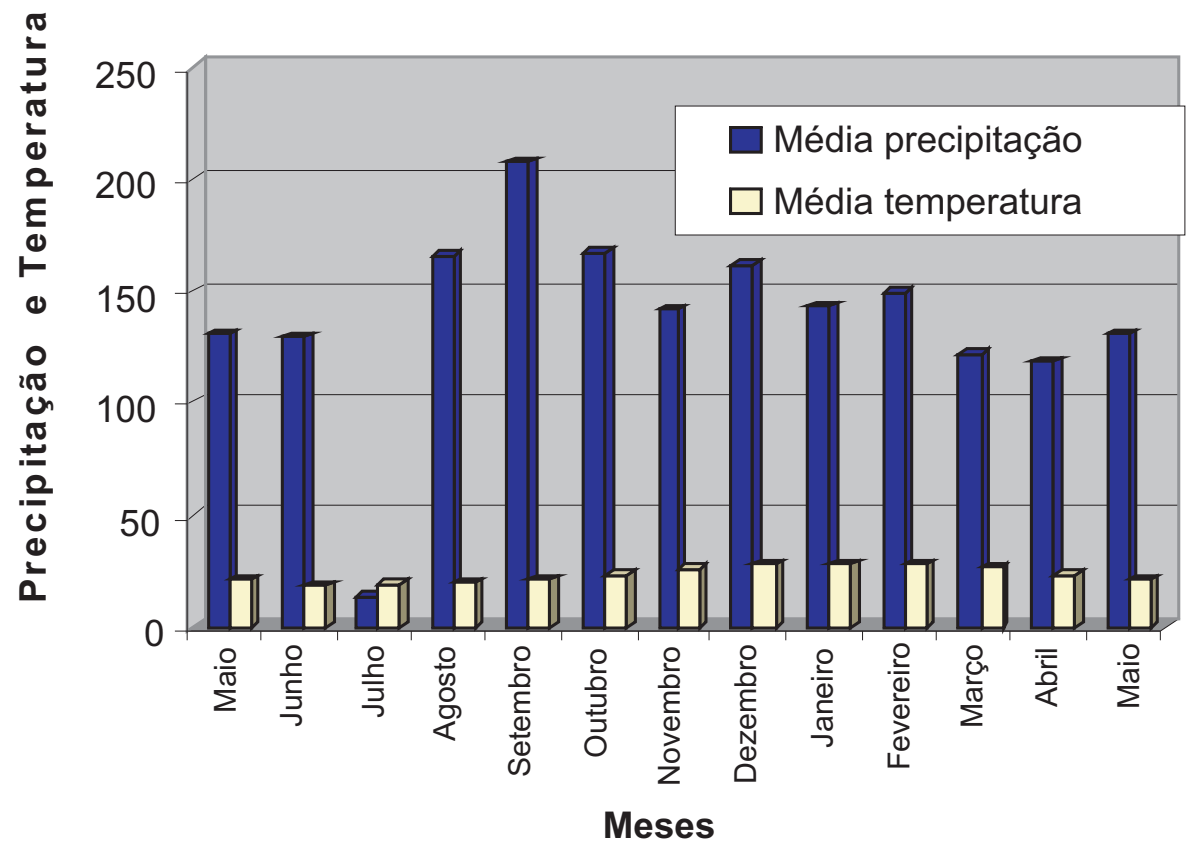

Figura 1. Média da precipitação pluviométrica na região de Passo Fundo de julho de 2005 a junho 2006. Fonte: EMBRAPA (1994). 
Pela classificação de Köppen (1948), o clima do local de estudo é descrito como subtropical úmido, com chuvas bem distribuídas durante o ano e temperatura do mês mais quente superior a $22^{\circ} \mathrm{C} \mathrm{e}$ a do mês mais frio oscilando entre 3 e $10^{\circ} \mathrm{C}$ (média anual de 16,9 a $18,4^{\circ} \mathrm{C}$ ) o que pode ser comprovado na Figura 2. O regime pluviométrico oscila entre 1575 e $1986 \mathrm{~mm}$ anuais, com a umidade relativa variando de 73 a 79\% (EMPRESA BRASILEIRA DE PESQUISA AGROPECUÁRIA - EMBRAPA, 1994). Estas condições ambientais são favoráveis ao desenvolvimento do $H$. contortus que foi o parasita mais prevalente neste estudo. As mudanças climáticas que vem ocorrendo ao longo dos anos podem interferir na epidemiologia do $H$. contortus conforme demonstrado no trabalho, uma vez que este parasita ocorreu em todas as estações avaliadas. Nieto et al. (2003) também encontraram maior prevalência deste parasita durante os períodos de julho a outubro de 1998 e de janeiro a abril de 1999 , mostrando que a distribuição deste parasita está diretamente ligada às alterações climáticas.

$\mathrm{O}$ custo de aplicação de fármacos foi menor nos grupos 3 e 4 , em função de que apenas 1,05\% das borregas e 2,63\% das ovelhas Famacha ${ }^{\circledR}$ necessitaram de controle parasitário, quando comparado ao grupo tradicional. A redução total do custo de aplicação de fármacos foi de $90,39 \%$ e 90,93\%, para borregas e ovelhas, respectivamente. No Paraná, foi relatado que, após a utilização desse método durante um período de 120 dias, foi possível reduzir em 79,5\% as aplicações com medicação antiparasitária em ovinos (MOLENTO; DANTAS, 2001). No presente trabalho ocorreram oito óbitos, quatro para borregas, sendo três no grupo Famacha e um no grupo tradicional, e quatro óbitos para ovelhas, um para o grupo Famacha e três para o grupo Tradicional. Entre as causas de mortalidade estão: acidentes de manejo, pneumonia, infecções secundárias, choque elétrico e procedimento cirúrgico. Nenhum dos óbitos ocorreu em função de infecções helmínticas.

\section{Conclusão}

O presente estudo permitiu concluir que as diferentes categorias de animais não apresentaram variações no peso vivo quando comparados os métodos tradicional e Famacha ${ }^{\circledR}$ no controle de nematódeos gastrintestinais. Com relação a variável OPG não houve diferença estatística a exceção da estação da primavera. Houve uma redução no custo de dosificações com a utilização do método Famacha ${ }^{\circledR}$ quando comparado ao método tradicional.

\section{Agradecimentos}

Aos funcionários do CEPAGRO que auxiliaram nas tarefas de campo.

\section{Referências}

ADANS, D. B. Changes in blood leukocytes, bone marrow and lymphoid organs in sheep infected with Haemonchus contortus. International Journal for Parasitology, Oxford, v. 11, n. 4, p. 309-317, 1981.

AMARANTE, A. F. T.; BARBOSA, M. A.; OLIVEIRA, M. A. G.; CARMELLO, M. J.; PADOVANI, C. R. Efeito da administração de oxfendazol, ivermectina e levamisol sobre os exames coproparasitológicos de ovinos. Brazilian Journal of Veterinary Research and Animal Science, São Paulo, v. 29, n.1, p. 31-38, 1992.

BRAGA, R. M. Sobrevivência de larvas infectantes de nematódeos gastrintestinais de bovinos, sob condições naturais. Revista Brasileira de Medicina Veterinária, Rio de Janeiro, v. 8, p. 186-188, 1986.

CARNEIRO, R. D. C.; SENO, M. C. Z.; RODRIGUES, C. F. C.; LEINZ, F. F.; BIANCHINI, D. Estudo da infecção helmíntica em cordeiros Suffolk submetidos a dois sistemas de terminação. Ciências Agrárias, Teresina, v. 27, n. 3., p. 489-496, 2006.

COLDITZ, I. G.; WATSON, D. I.; GRAY, G. D.; EADY, S. J. Some relationship between age, immune responsiveness and resistance to parasites in ruminants. International Journal for Parasitology, Oxford, v. 26, n. 8-9, p. 869-877, 1996.

ECHEVARRIA, F. A. M. Doenças parasitárias de ovinos e seu controle. In: SIMPÓSIO PARANAENSE DE OVINOCULTURA, 3., 1986, Guarapuava. Anais... Londrina: IAPAR, 1988. p. 46-47. 
ECHEVARRIA, F. A. M.; BORBA, M. F. S.; PINHEIRO, A. C., WALLER, P. J., HANSEN, J. W. The prevalence of anthelmintic resistance in nematode parasites of sheep in Southern Latin America: Brasil. Veterinary Parasitology, Amsterdam, v. 62, n. 3-4, p. 199-206, 1996.

EMPRESA BRASILEIRA DE PESQUISA AGROPECUÁRIA - EMBRAPA. Macrozoneamento agroecológico e economico. Porto Alegre: EMBRAPA/ CNPT, 1994.

GORDON, H.M.; WHITLOCK, H.V.A new technique for counting nematode eggs in sheep faeces. Journal of Council of Science and Industry Research, Australia, v.12, n.1., p. 50-52, 1939.

KAWANO, E. L.; YAMAMURA, M. H.; RIBEIRO, E. L. A. Efeito do tratamento com anti-helmíntico em cordeiros naturalmente infectados com helmintos gastrintestinais sobre os parâmetros hematológicos, ganho de peso e qualidade de carcaça. Arquivos da Faculdade de Veterinária, Porto Alegre, v. 29, n. 2, p. 113-121, 2001.

KÖPPEN, W. Climatologia. México: Fundo de Cultura Econômica, 1948.

LANUSSE, C.; PRICHARD, R. Relationship between pharmacological properties and clinical efficacy or rumiants anthelmintics. Veterinary Parasitology, Amsterdam, v. 49, n. 2-4, p. 123-158, 1993.

MOLENTO, M. B.; DANTAS, J. C. Validação do guia Famacha ${ }^{\circledR}$; para diagnóstico clínico de parasitoses em pequenos ruminantes no Brasil: resultados preliminares. In: ENCONTRO INTERNACIONAL SOBRE AGROECOLOGIA E DESENVOLVIMENTO RURAL SUSTENTÁVEL. 1., 2001, Botucatu. Anais... Botucatu: UNESP, 2001. v. 1, p. 58.

MOLENTO,M.B.;TASCA, C.; GALLO,A.;FERREIRA, M.; BONONI, R.; STECCA, E. Método Famacha ${ }^{\circledR}$ como parâmetro clínico individual de infecção por Haemonchus contortus em pequenos ruminantes. Ciência Rural, Santa Maria, v. 34, n. 4, p. 1139-1145, 2004.
NIETO, L. M.; MARTINS, E. N.; MACEDO, F. A. F.; ZUNDT, M. Observações epidemiológicas de helmintos gastrintestinais em ovelhas mestiças manejadas em pastagens com diferentes hábitos de crescimento. Ciência Animal Brasileira, Goiânia, v. 4, n. 1, p. 45-51, 2003.

O'SULLIVAN, B. M.; DONALD, A. D. A field study of nematode parasite populations in the lactating ewe. Parasitology, Cambridge, v. 61, n. 2, p. 301-315, 1970.

RAMOS, C. I.; BELLATO, V.; SOUZA, A. P.; AVILA, V. S.; COUTINHO, G. C.; DALAGNOL, C. A. Epidemiologia das helmintoses gastrintestinais de ovinos no Planalto Catarinense. Ciência Rural, Santa Maria, v. 34, n. 6, p. 1889-1895, 2004.

ROBERTS, F. H. S.; SULLIVAN, P. J. Methods for egg counts and larval cultures for strongyles infecting the gastro-intestinal tract of cattle. Australian Journal of Agricultural Research, Victoria, v.1, n.1, p. 99-102, 1950.

UENO, H.; GONÇALVES, P. C. Manual para diagnóstico das helmintoses de ruminantes. 4. ed. Tokio: Japan International Cooperation Agency, 1998. 143p.

VIEIRA, L. S.; CAVALCANTE, A. C. R. Alternativas de controle da verminose gastrintestinal dos pequenos ruminantes. Sobral: EMBRAPA/CNPC, 2003. (Circular Técnica, 29).

VAN WYK, J. A.; MALAN, F. S.; BATH, G. F. Rampant anthelmintic resistance in sheep in South Africa - what are the options? In: WORKSHOP MANAGING ANTHELMINTIC RESISTANCE IN ENDOPARASITES. INTERNATIONAL CONFERENCE OF THE WORLD ASSOCIATION FOR THE ADVANCEMENT OF VETERINARY PARASITOLOGY, 16., 1997, Sun City. Proceedings... Sun City, South Africa: WAAP, 1997. p. 51-63.

WALLER, P. Anthelmintic resistance. Veterinary Parasitology, Amsterdan, v. 72, n. 3-4, p.391-412, 1997. 
